\title{
Design and Application of Wind Power Peak Control Technology
}

\author{
Songyi Zhu1, Xinhua Deng2, Shuguang Meng3, Fuqiang Tang1 \\ ${ }^{1}$ NARI Technology Co., Ltd., Nanjing, China \\ ${ }^{2}$ China Power Investment Corp Chong Qing Branch, Chongqing, China \\ ${ }^{3}$ Shandong Bing Hua Group Incorporated Company, Binzhou, China \\ Email: eechoo125@163.com
}

Received May 2014

\begin{abstract}
Wind power control technology is an important part of intelligent control in wind farms. By the automatic calculation and implementation of control strategy, problems such as imprecise of manual control scheduling, slow adjust rate, heavy workload, etc. have been solved. It can improve the capacity of wind power grid, and it also has the important meaning to the safe and stable operation of power grid. This paper introduces wind power control system from certain aspects such as control mode, control principle, and so on.
\end{abstract}

\section{Keywords}

Wind Power Peak Control, Active Power Control

\section{Introduction}

For years, with the development of new energy technology, wind power generation technology is widely used in the new energy power generation field precisely because of its abundant resources, non polluting and renewable characteristics. But since wind power generation has some unusual features such as intermittent, random, anti peaking, etc., the safety and the stability of power grids may be greatly influenced after its large scale access.

Considering these situations, the publications of "China State Grid Corp wind power technology" (GB/Z199632009) proposed that wind farms should have the ability to regulate active power, and to control their active power output according to the commands from power grid dispatching department. In order to achieve this goal of power control, wind farms need to increase or upgrade their active power control system, so as to receive and automatically execute the active control commands sent from dispatching department, and to ensure that the maximum wind power numerical value and active power change value does not exceed the given value which is sent from the dispatching department [1].

Wind power peak control system is just such an effective power control system designed and developed to meet all these purposes. 


\section{System Overview}

\subsection{System Function}

In accordance with the command from power grid dispatching department, the wind power peak control system is designed to dynamically adjust the active power output of the wind farm, by the methods of wind turbines pitch control or stop-start control. And all these operations must be under the condition ensuring the service life of wind turbines, as well as reducing the wind turbine faults. Specific functions include:

1) Send effective power control commands to wind farms.

2) Communicate with wind power predict system; share and exchange the data to achieve the goal of constant power control.

3) Wind farm grid connection remote control

4) Automatically control the maximum output of the related wind farms, according to the stability requirements of the sections

5) Monitor the system frequency, control wind farm output and adjust power grid frequency for the longterm.

\subsection{Control Method and Control Principle}

There are three control methods involved in wind power peak control, that is: advance peak power control, normal peak power control and emergency peak power control.

Advanced peak power control is also known as pre power control. It sends control command according to the load regulating capacity which is calculated ahead by the system. This control mode is implemented through the following methods: wind turbines pitch; wind turbines start or stop.

Normal peak power control achieves the goal of peak control according to the real-time calculation of the load capacity, this control mode is implemented through the following methods: 1) Wind turbines pitch; 2) Wind turbines start or stop; 3) Remove wind farm $35 \mathrm{kV}$ feeder in optimization.

Emergency peak power control is supposed to deal with emergency situation; this control mode is implemented through the following methods: remove wind farm $35 \mathrm{kV}$ feeder in optimization.

Wind power peak control system is worked on the basis of three principles, 1 st is fair distribution of wind power, 2nd is minimum the wind power energy loss, and 3rd is no damage of the wind turbines at any time. Specifically, first of all, we should ensure as far as possible that each wind farm to get equal opportunities to access the wind power plan. And then It is recommended that the resources of each wind farm are optimally distributed, various information in each wind farm is fully opened, and is transparent to each other [2]. On this basis, maximum output of each wind farm and minimum damage of wind turbines may be expected and achieved.

\subsection{Control Mode}

There are two different control modes in wind power peak control: open loop mode and close loop mode. In open loop mode, calculation results of peak control strategy will be fed back to the users through the terminal, and after the users' examination and verification, peak control command is manually sent ultimately. In closed loop working mode, peak control strategy is calculated automatically by the system while control commands are also issued automatically by the system. In this mode, not a single link of the peak control process needs manual participation. These two kinds of control mode can be configured flexibly in order to meet different needs and is suitable for different occasions.

\section{Control Process}

During the actual operation, wind power peak control system computes in real time the maximum acceptance capacity of current power grid, according to the constraints of power peaking requirements. It also computes the wind power generation scheme for each wind farm in accordance with the change of wind power receptivity, the current output power of wind farms and the proposed output numerical value of wind power prediction system. Afterwards, the scheme motioned before is sent to the wind farms and the active power control devices in each wind farm control the wind turbines according to the planned value. The detail control process is shown in Figure 1. 


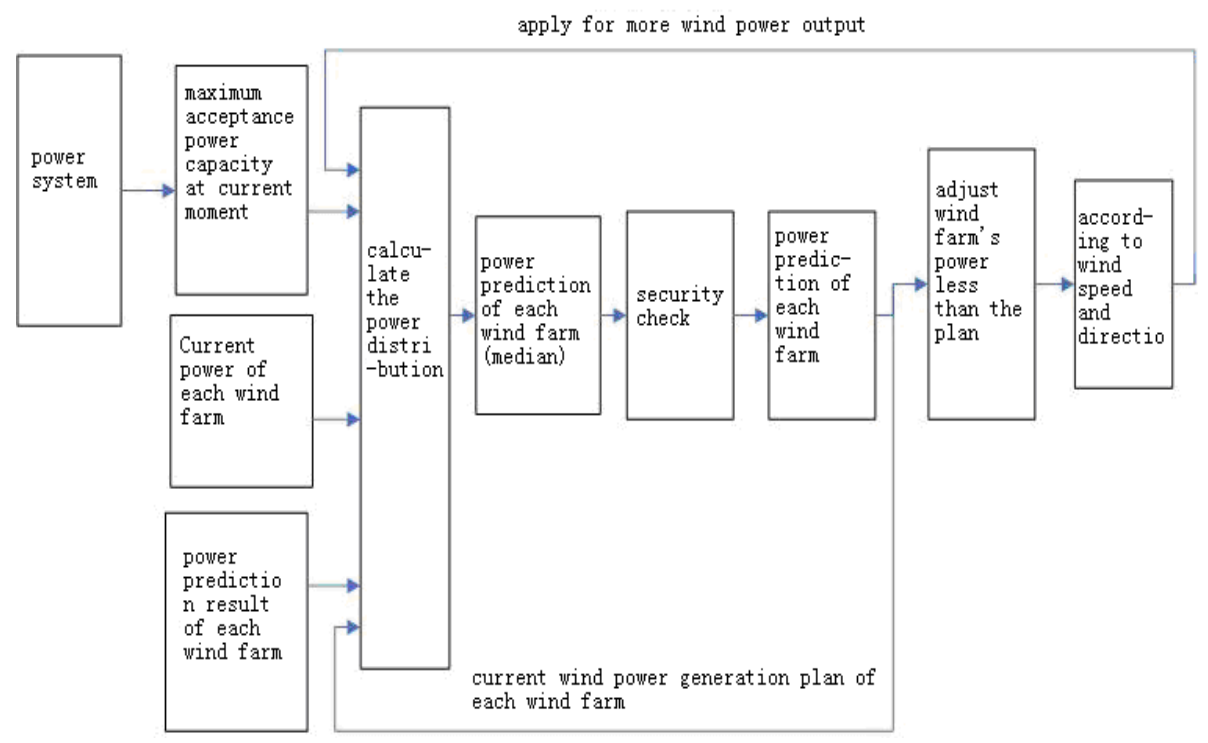

Figure 1. Detailed control process.

\section{Open-Loop Control Mode}

Open-loop control mode is also named as power plan manually calculate mode. In this mode, the system automatically calculates the control plan of each wind farm, the calculate result is not directly sent to wind farms, but is displayed on the terminal of dispatchers for their reference uses. The specific operation can be divided into "wind power limit mode” and “wind power remove limit mode”.

\subsection{Wind Power Limit Mode}

\subsubsection{Peak Power Control}

Wind power control system not only supports users to set wind power plan numerical values (maximum output power) for specified wind farm, but also allow users to set the total amount of adjustment for all wind farms, New wind power plan numerical values are calculated automatically by the wind power control system.

Users can specify which wind farm is selected in the power control procedure. Meanwhile, it is required for users to give out the evaluation index of each wind farm. The control operation is under the principle of fair and just, and according to the assessment results, fast and efficient calculation of wind power output plan is expected. When the total adjustment amount is small, the wind farms which have poor assessment will have the priority to adjust first, otherwise all wind farms are involved into the calculation.

There are two methods to calculate power generation plan value in wind power control procedure. The first method is to consider the wind farm assessment index, keep the ratio of new power plan adjustment numerical values closing to the wind farm Installed capacity. In this circumstance, the calculation method of the power generation plan can be realized by the following formula:

$$
\begin{aligned}
& \min \left(\sum_{i \in N} \Delta P i-\Delta P s e t\right) \\
& \text { s.t. } \frac{P i-K i \cdot \Delta P i}{P i, \max }=\frac{P j-K j \cdot \Delta P j}{P j, \max } \quad i, j \in N
\end{aligned}
$$

In above formula, $\Delta$ Pset is total wind power control amount, $N$ is number of wind farms which is involved in the control process, $\mathrm{Pi}$,max is wind farms' total power capacity, $\mathrm{Pi}$ is the current output power of wind farm, $\mathrm{Ki}$ is evaluation index results for all wind farms (the results have been normalization yet), $\Delta P i$ is the adjust control value, and through $\Delta P i$, new power generation plan $P i, g$ can be calculated by formula (2):

$$
P i, g=P i-\Delta P i
$$

The second method of calculating power generation plan is to consider the wind farm evaluation index results, 
calculate the new power generation plan according to the ratios of the present power output. The formula is:

$$
\begin{aligned}
& \min \left(\sum_{i \in N} \Delta P i-\Delta P s e t\right) \\
& \text { s.t. } \frac{\Delta P i}{\Delta P j}=\frac{P i / K i}{P j / K j} \quad i, j \in N
\end{aligned}
$$

\subsubsection{Section Power Control}

In order to reduce the power load of specified section, users are allowed by wind power control system not only by specifying the wind power plan of the selected wind farms one by one, but also by setting total amount of adjustment value of specified sections. New wind power generation plan of the related wind farms or wind farm sections can be calculated by the wind control system automatically.

The object of peak wind power control is all wind farms in the power grid, while the object of section wind power control is the associated wind farms which had been pre specified. By configuration, users can divide the wind farms into several wind power control subsets (section), and control the power of corresponding sections by the method of adjusting the power generation plan of each subset.

Section wind power control has the same calculation methods as peak wind power control, because wind farms involved in Section power control are the subset of those involved in peak power control.

\subsubsection{Flow Chart of Wind Power Limit Mode}

The flow chart of wind power limit mode is shown in Figure 2.

\subsection{Wind Power Remove Limit Mode}

\subsubsection{Principle of Removing Limit Control Operation}

In order to balance the load growth or increase wind power electricity of specified sections, it is supported by wind power control system to allow users to cancel the power limit plan for wind farms by selecting wind farms manually. It is also supported by the system to cancel the power limit plan by setting the total control amount, and then selecting wind farms automatically according to that amount, so as to allow wind farms to increase the power output as far as possible.

When remove the wind power restrictions, users should consider comprehensively about the wind farm evaluation index result and the beginning time of wind farm restrictions. After that, wind farms should be selected and the power limit should be canceled one by one. Then, waiting for a period of time, calculate the power change before and after the control. If the power change is greater than the total adjustment amount, the control operation can be terminated, otherwise operation continues by selecting more wind farms.

\subsubsection{Flow Chart of Wind Power Remove Limit Mode}

The flow chart of wind power remove limit mode is shown in Figure 3.

\section{Close Loop Mode Control}

In order to track the change of electrical power grid, wind power peak control system should also have the function of calculating and sending the power plan of wind farms automatically. This is called the close loop control mode.

Close loop control mode, namely the power schedule automatic calculation mode. Here is the workflow of this mode: Under user's permission, wind power peak control system runs all calculation periodically, tracks the power grid operation state automatically, without considering various prediction results, gives out the real-time calculation result of current power plan to meet the current power load, and then directly send control commands to the relevant wind farms.

The calculation principle of close loop wind power control model is: first, calculate power system standby load $\eta s$, when $\eta s$ is less than the lower bound value $\varepsilon 1$, calculate the required adjustment value of improving the system load, then calculate the wind farms which are needed to remove the output limit. At the same time, adjustability, priority and other factors should be considered, so as to maintain the output power of hydropower and thermal reducing evenly. 


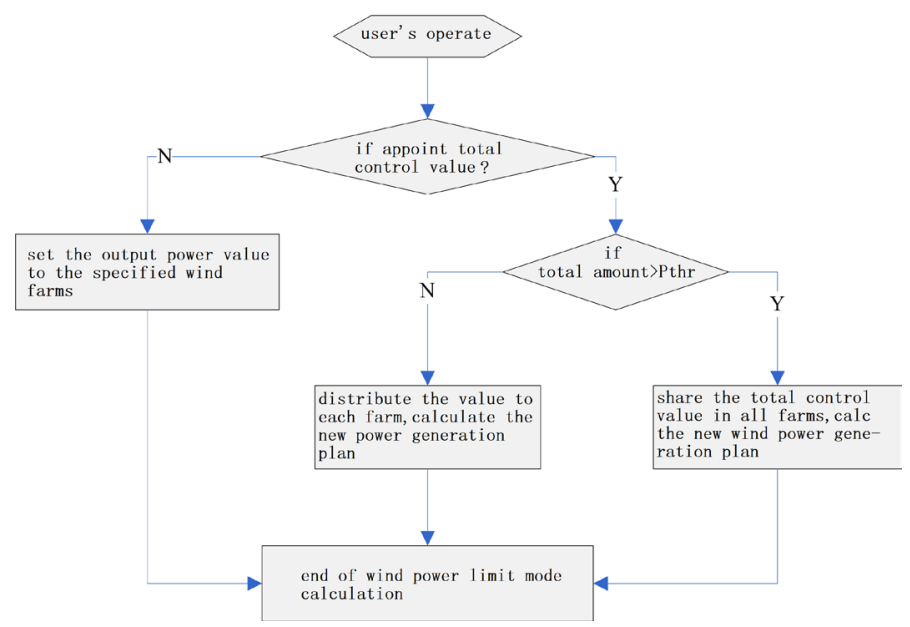

Figure 2. Power limit mode flow chart.

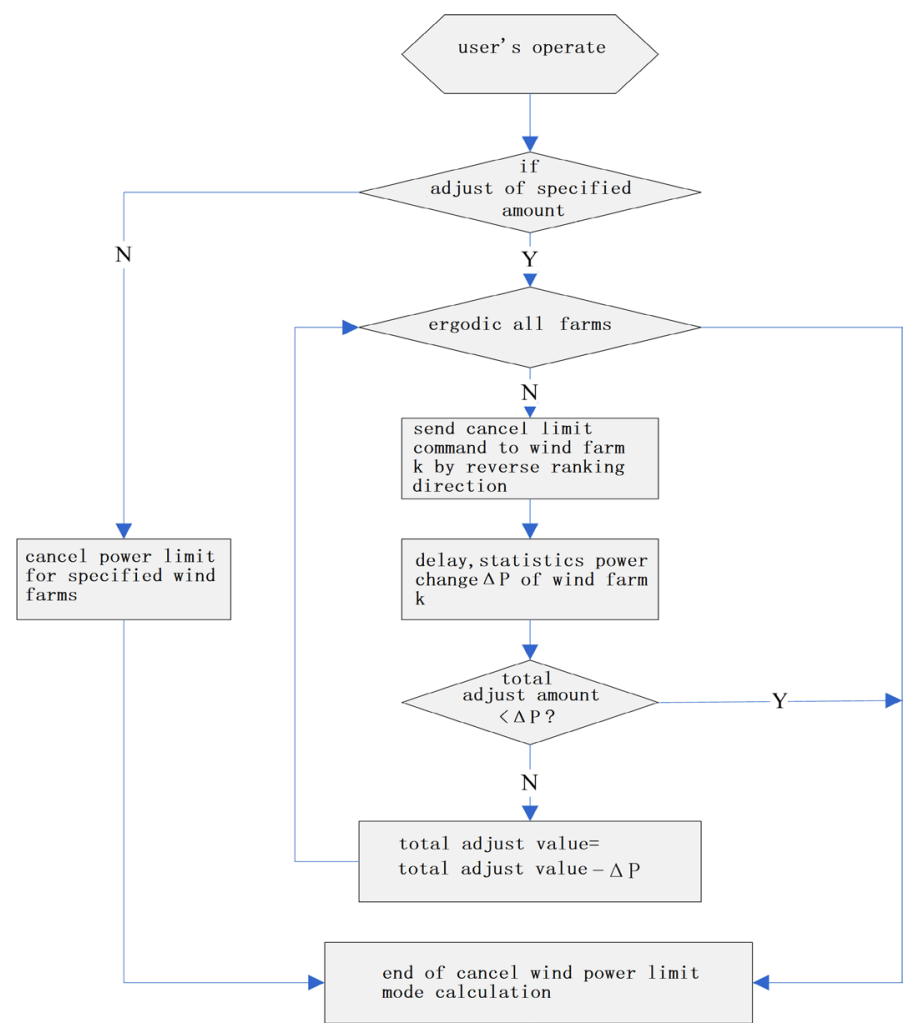

Figure 3. Remove power limit mode flow chart.

\section{Conclusions}

With the increasing development of wind power technology and fast growing of wind power capacity, controllable operation, as well as has been incorporated into the large power grid dispatching system, has gradually become the development trend of large scale wind farms [3]. Among them, the active power control is a key technology of controllable operation system in wind farms. The active power control strategy of wind farm is realized through coordinating the various kinds of wind turbines in one wind farm.

Wind power peak control system substitute the dispatcher's real-time control of wind power, reduces the frequent business contacts and the complicate calculation between dispatchers and wind farms. It solves many problems that are encountered in traditional manual control scheduling, such as imprecise, slow adjust speed, 
heavy workload, etc. Meanwhile, wind power peak control system also maximizes the use of grid resources, improve the power capacity of wind farms, and improve the power output of wind farms too. It has an important significance to strengthen the management and control ability in wind farms.

\section{References}

[1] China State Grid Corp (2009) Provisions of the Wind Power Grid Technology. Revised Edition, GB/Z19963.

[2] Dexue, Y. and Cong, Z.H. (2011) Research and Practice of AGC System in Wind Farm. Northeast Electric Power Technology, 4th Period.

[3] Hui, J. and Gu, X. (2010) Research on Centralized Power Control Strategies for Large Wind Farms. East China Electric Power, 6th Period. 